\title{
COLOR IMAGES
}

\section{DOMINIQUE LAFON AND TAHIANA RAMANANANTOANDRO}

Ecole des Mines d'Alès - Centre des Matériaux de Grande Diffusion - E2PSM 2 avenue Paul Angot 64053 PAU Cedex 09, France e-mail: Dominique.lafon@ema.fr, Tahiana.Ramananantoandro@ema.fr (Accepted September 25, 2002)

\begin{abstract}
The goal of this article is to present specific capabilities and limitations of the use of color digital images in a characterization process. The whole process is investigated, from the acquisition of digital color images to the analysis of the information relevant to various applications in the field of material characterization. A digital color image can be considered as a matrix of pixels with values expressed in a vector-space (commonly 3 dimensional space) whose specificity, compared to grey-scale images, is to ensure a coding and a representation of the output image (visualisation, printing) that fits the human visual reality. In a characterization process, it is interesting to regard color image attributes as a set of visual aspect measurements on a material surface. Color measurement systems (spectrocolorimeters, colorimeters and radiometers) and cameras use the same type of light detectors: most of them use Charge Coupled Devices sensors. The difference between the two types of color data acquisition systems is that color measurement systems provide a global information of the observed surface (average aspect of the surface): the color texture is not taken into account. Thus, it seems interesting to use imaging systems as measuring instruments for the quantitative characterization of the color texture.
\end{abstract}

Keywords: color, color image, color texture, colorimetry, material, wood.

\section{INTRODUCTION}

Color image analysis is a field of growing importance that concerns a wide range of applications. Indeed, important technical progress in terms of color sensors and computational capabilities allow a significant decrease in the cost of color imaging systems. Color images play an important role in the field of simulation (representation of realistic visual aspects of materials in some prototyping process, and more generally in virtual reality scenes), and in the field of material characterization at the microscopic and macroscopic scales. A new field of investigation is currently being developed that concerns psychovisual properties of materials, considered as «use properties» in the industrial context.

In a characterization process, color, just as any other attributes such as shape and size, can be taken as a marker for distinguishing an object, or more generally a phase. In this case, color will be involved in the pre-processing and segmentation steps. Color can also be regarded as a psychovisual property to be quantified. In this case, multi-component images will be analysed in terms of statistic and spatial distributions of color vectors (Fig. 1).

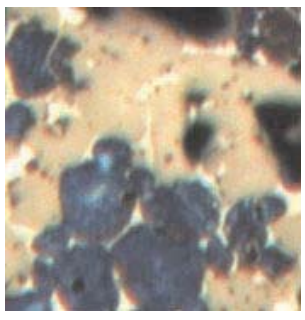

(a) color as a result of acid etching

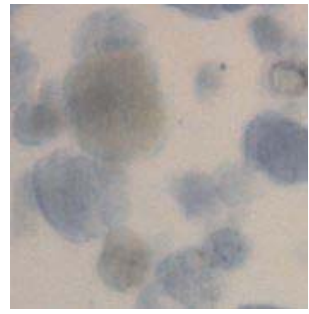

(b) color is directly linked to crystal system

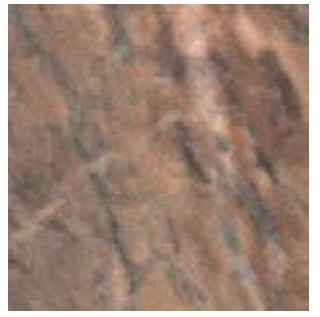

(c) Color is the studied "spatial" property (color texture of a marble)

Fig 1. Digital imaging and color - color as a marker ( $a, b)$; color as a property (c). 
Color is a complex property of importance in such scientific fields as physics, chemistry, physiology, psychology, ... At present, no set of parameters exist that can quantify all the color properties. For instance, it is not possible to study perceptual color differences by using a RGB (Red, Green, Blue) color coding. Depending on his goal, a color-user has to choose among several different color approaches; a very important point is to identify, and describe, the specific color property relevant to a particular characterization process (specificity can be related to physico chemical properties of the material; it can also be related to a specific observation context). A crucial point in color image analysis is to choose the appropriate acquisition system and to express color in the correct color coding system.

In this paper, we present color as a material property resulting from complex interactions. We approach the problem of color characterization through two point of views: a psycho physiological approach, and a colorimetric approach. Then, we give an overview of the specific constraints relating to color imaging systems, color image processing and analysis. The selected example is used to emphasize the very important influence of existing correlations between color attributes on color analysis and understanding.

\section{SOME GENERALITIES ABOUT COLOR}

A good comprehension of what color is, constitutes an essential prerequisite to color control in digital imaging. Color is only defined as long as a light stimulus (electromagnetic wave with wavelength falling between 380 and $780 \mathrm{~nm}$ ) excites the human eye photoreceptors. Color exists only through the interaction between a luminous radiation and a vision system. A vision system must be able to receive information and translate it in terms of sensation involved in the perception of the environment (Fig. 2).

The incident stimulus results from the interaction between the object surface and the light emitted from a given source. A stimulus $S(\lambda)$ is defined as the result of a convolution product between a spectral power distribution of light source $\mathrm{I}(\lambda)$ and the object spectral reflectance $R(\lambda)$ :

$$
S(\lambda)=I(\lambda) \cdot R(\lambda) \text { with } \lambda=\text { wavelenght } .
$$

Optical phenomena involved in light / material interaction are transmission, absorption and reflexion (Sève, 1996). Material properties influencing these phenomena are of crystallographic and chemical nature (Wyszeski and Stiles, 1982).

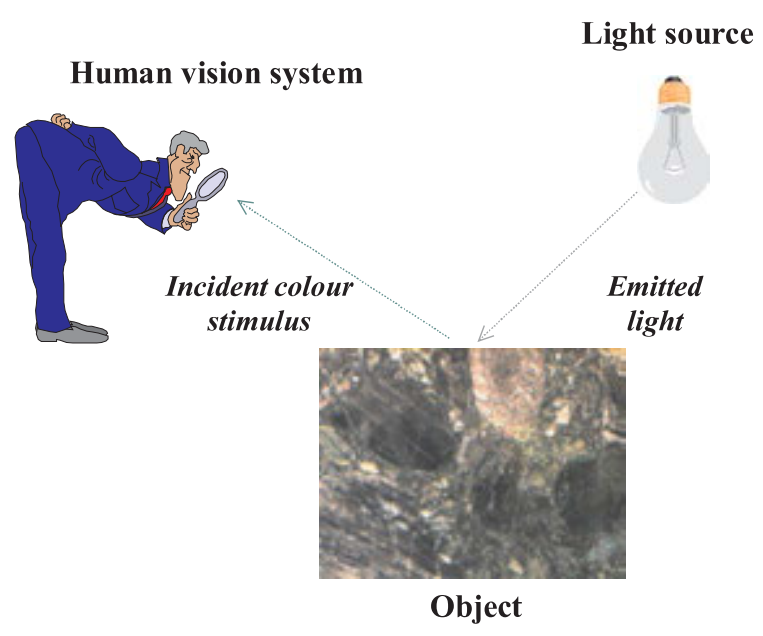

Fig. 2. Color Vision - Object-light-sensor triplet.

Roughness of the surface plays also a significant role as well as the patterns of the different components of the superficial layer:

- The same object will have different spectral reflectances depending on its roughness (Fig. 3). If one wishes to use color in chemical and/or crystallographic identification, it is very significant to ensure that the surface state of different samples of the same product remains unchanged during color image acquisition.

- Color of a pulverulent material is a complex property depending on individual properties of particles and on collective properties of the entire population (Bozec-Garay, 2000). Particle shape and size, particle spatial distribution in the superficial layer play a very important role in the aspect of the pulverulent material.

Eq. 1 highlights the influence of illumination in color perception. The example of Fig. 4 illustrates the importance of taking into account the spectral characteristics of the light source in the formation of the color image. A spectrum variation can lead to a noticeable change in the object aspect. Thus, two images acquired under different lighting conditions will not be easily comparable if the image context is the only available information. On the one hand, this can constitute a significant handicap when developing an automated segmentation protocol. On the other hand, it is possible to optimize object illumination in order to highlight some interesting color or spectral characteristics, or hide some irrelevant color information. 


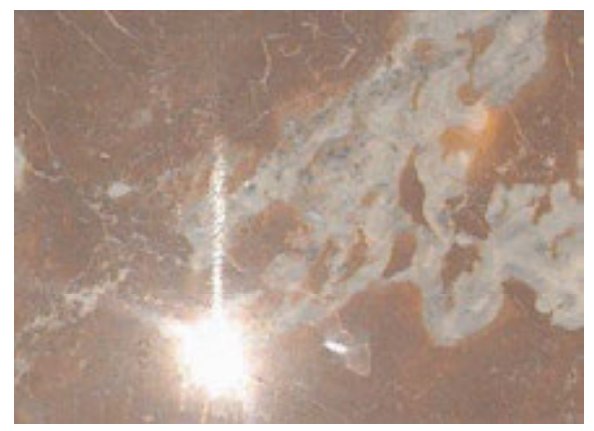

Polished surface

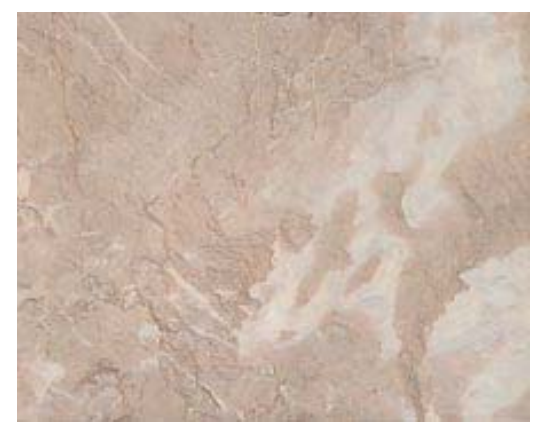

Rough surface

Fig. 3. Influence of the surface finish of a material on its visual aspect (same marble, same surface illumination, but different roughness).

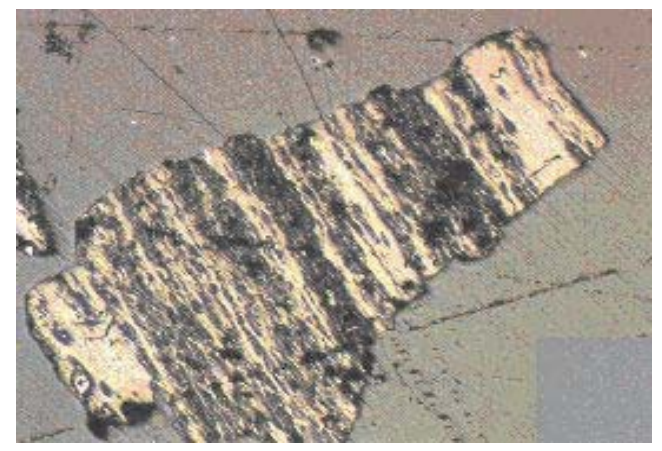

Polished section - Sunny day

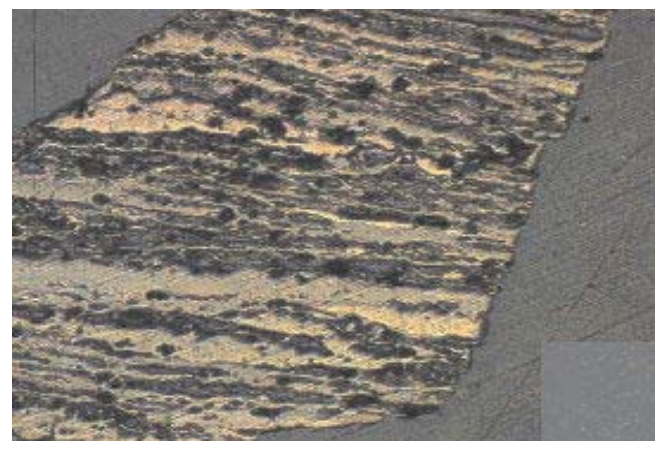

Polished section - Cloudy weather

Fig. 4. Illumination influence on the human perception of color (coal under microscope).

\section{TWO DIFFERENT APPROACHES TO COLOR QUANTIFICATION}

Color can be described as a spectral power distribution in the visible part of the electromagnetic spectrum. This description is not directly linked to the human description of color sensation. Depending on the (color) application, it can be useful to speak about physical color, psycho physical color, perceived color, or cognitive aspects of color.

In this section, we will present two different approaches to color characterization: the first one is based on a psycho physiological approach; the second one corresponds to an industrial problematic concerning the measurement and the prediction of colors.

\section{Psycho physiological aspect of color}

The human eye does not analyze the inpiging color stimulus according to a uniform sampling process of the spectrum: the spectral power distribution of the stimulus $S(\lambda)$ is not sampled by the eye with a constant step. It is significant to examine the working process of the human eye; this constitutes a useful prerequisite in the design of color spaces related to psycho physiological and cognitive aspects of color; furthermore this makes the evaluation of a frame grabbing system potentialities possible in terms of color information quantification.

The color stimulus excites different types of photoreceptors located in the superficial part of the retina (Sève, 1996). These specialized nerve cells contain photosensitive pigments that are able to transform light energy in an electrical (nervous) signal. There are two types of photoreceptors that have a non-uniform spatial distribution at the surface of the retina (Roorda and Williams, 1999):

- Rods are reserved for the perception of light intensity. Their spectral sensitivity vary with the wavelength. The sensitivity curve is a bell-shaped curve with a maximum at $498 \mathrm{~nm}$ in the case of photopic vision. At low light level (scotopic vision) only rods are active with a higher maximum luminous efficiency $(555 \mathrm{~nm})$. The light sensitive pigment in rods is called rhodopsin.

- Cones are less sensitive to light intensity; they are reserved for color perception and are only efficient for diurnal vision. These cells are concentrated in the central part of the retina called fovea. There are three different types of 
cones: L cones have a peak sensitivity at 564 nanometres; $M$ cones have a peak sensitivity at $533 \mathrm{~nm}$; S cones have peak sensitivity at $437 \mathrm{~nm}$ ) (Bowmaker and Dartnall, 1980). Each type has a particular visual pigment (erythrolabe for $\mathrm{L}$ cones, chlorolabe for $\mathrm{M}$ cones and cyanolabe for $\mathrm{S}$ cones) but there is a noticeable overlap between cone sensitivities.

Fig. 5 presents the estimated spectral sensitivities of photoreceptors. As it can be seen in Fig. 5, there is a marked overlap between $\mathrm{L}$ and $\mathrm{M}$ cones: thus $\mathrm{L}$ and $\mathrm{M}$ responses to a stimulus will be highly correlated. The $\mathrm{S}$ sensitivity is definitively lower than the $\mathrm{M}$ and $\mathrm{L}$ sensitivities (low $\mathrm{S}$ density in the fovea). Thus, the spatial resolution is much lower for blue and violet than for other colors.

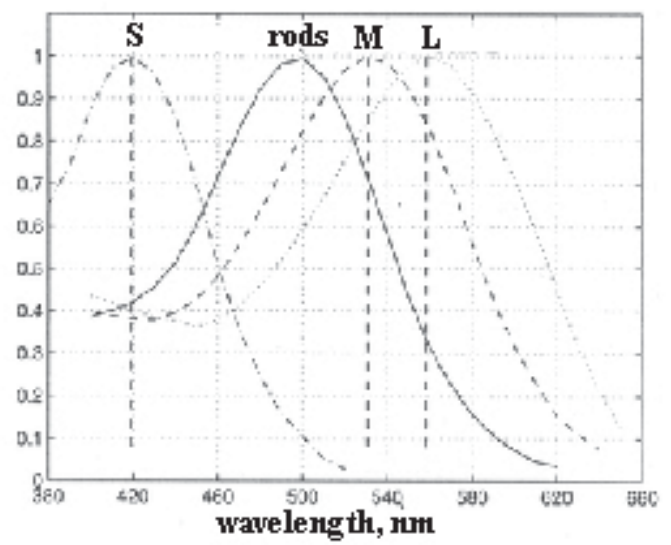

Fig. 5. Estimated spectral sensitivity of the photoreceptors of the human eye: $l(\lambda), m(\lambda), s(\lambda)$ and $r(\lambda)$.

Cone responses to a stimulus $S(\lambda)$ are obtained by integration according to Eq. 2:

$$
\left\{\begin{array}{c}
1=\int_{\lambda \min }^{\lambda \max } 1(\lambda) \cdot S(\lambda) \cdot d \lambda \\
\mathrm{m}=\int_{\lambda \min }^{\lambda \max } \mathrm{m}(\lambda) \cdot \mathrm{S}(\lambda) \cdot \mathrm{d} \lambda \\
\mathrm{s}=\int_{\lambda \min }^{\lambda \max } \mathrm{s}(\lambda) \cdot \mathrm{S}(\lambda) \cdot \mathrm{d} \lambda
\end{array}\right.
$$

A good understanding of the behavior of the L, M and $\mathrm{S}$ cones is helpful in the design and the calibration of a color image sensor.

Cone responses are transmitted to the cerebral cortex via a complex process that involves interactions between the different signals. There is no direct relation between cone activity and sensation. Transmitted nervous signals are transformation of the cones initial responses. These transformations consist in weighting, combining and multiplexing the different signals (De Valois, 1965).

The final combination in the brain seems to generate an opponent color system (three channels: an achromatic black/white channel and two chromatic red/green and blue/yellow channels) (Faugeras, 1979; Bedat, 1998). This corresponds to the Herring's Opponent Theory (1878). Then, a neural image of an object is formed in the cerebral cortex by conscious reasoning (through explicit knowledge). A person will describe the color appearance of a plain surface according to three attributes that are not connected in a simple way to the stimulus spectral power distribution, the hue, the luminance and the saturation. Thus, the operation of the human visual system leads to a nonuniform perception of luminance and chromatic attributes of color. In addition, two stimuli with very different spectral power distributions (metameric color stimuli) will induce the same color feeling because of spectral power distribution of light, or because of the objects spectral reflectance. Human visual system does not process the color stimulus linearly.

The human visual system generally deals with complex scenes. Perception processes involve an integration of the global characteristics of the scene. The perceived colors cannot be expressed simply through a local approach. The apparent color of an area is influenced by its neighborhood (Barnes et al., 1999; Longère et al., 2000). Furthermore, the human visual system is able to recognize colors independently of lighting conditions; it can also balance transparency effects (D'Zmura et al., 2000).

Actually, there does not exist any color quantification system taking into account all these phenomena.

\section{Colorimetry}

A basic hypothesis in colorimetry is that color stimulus can be measured and quantified. Colorimetry concerns industrial problems: how to compare the color of different plain samples? How to formulate a color (Judd and Wyszecki, 1975)?. Colorimetry considers only plain samples and it is a restrictive approach; it does not take into account the complex interactions discussed above.

Measurement and imaging devices work on the basis of the trichromatic properties of the human visual system (Sève, 1996). The trichromatic theory of color vision was proposed in 1801 by Thomas Young, and rediscovered by Hermann Von Helmholtz around 1852. This theory is based on the color 
matching experiments performed by Maxwell. The Young-Helmholtz theory and the Herring's opponentprocess theory complement each other. In the trichromatic approach, most of the color stimuli $S(\lambda)$ can be reproduced by the mixture (superposition of light, i.e. addition) of three independent monochromatic lights called primaries. It is possible to perform colormatching experiments using monochromatic test light for each wavelength (Fig. 6). For a given set of primaries, a set of three curves can be obtained. These color-matching functions are called $\bar{r}(\lambda), \bar{g}(\lambda), \bar{b}(\lambda)$. They correspond to the amount of each primary that would be required to match a defined amount of monochromatic light (test light) for a «normal» observer. Of course, color matching functions derived for different primary triplets would differ.

Fig. 6 shows the color matching functions corresponding to a set of red, green and blue primaries. The red color matching function shows a negative zone. Some monochromatic lights cannot be matched by any addition of these three primaries; in order to achieve a match, it is necessary to add a red light to the monochromatic test light. This problem arises for any set of physically realisable primaries.

Tristimuli values are equal to the amount of each primary needed to match a color.

Colorimetry is a color specification system developed by the Commission Internationnale de l'Eclairage (CIE) (Wyszeski and Stiles, 1982). Colorimetry aims at describing the interaction between color physical aspects and physiological characteristics of the human visual system. Its main goal is to determine if two stimuli will visually match when they are viewed under controlled lighting and viewing conditions. It is based on the following prerequisites:

- The set of primaries adopted in 1931, by the CIE, corresponds to three monochromatic lights with wavelengths of $700 \mathrm{~nm}, 546.1 \mathrm{~nm}$ and $435.8 \mathrm{~nm}$, respectively. It defines the behavior of the Standard Colorimetric Observer.

- Specific viewing conditions have been used to define the standard set of color-matching functions. They define very precisely how an object has to be measured. As said previously, lighting conditions play an important role in color perception. The CIE defines several illuminants which are specified in terms of the relative energy tabulated for each wavelength (all illuminants cannot be physically realized as a light source). The colorimetric values are not appearance descriptors. They much rather provide a tool for evaluating differences between two stimuli (only in case they are close).

- The (R, G, B) tristimuli values are obtained by summing over the visible spectrum the product of these three functions with $S(\lambda)$ :

$$
\left\{\begin{array}{l}
\mathrm{R}=\int_{\lambda \min }^{\lambda \max } \mathrm{r}(\lambda) \cdot \mathrm{S}(\lambda) \cdot \mathrm{d} \lambda \\
\mathrm{G}=\int_{\lambda \min }^{\lambda \max } \overline{\mathrm{g}}(\lambda) \cdot \mathrm{S}(\lambda) \cdot \mathrm{d} \lambda \\
\mathrm{B}=\int_{\lambda \min }^{\lambda \max } \overline{\mathrm{b}}(\lambda) \cdot \mathrm{S}(\lambda) \cdot \mathrm{d} \lambda
\end{array}\right.
$$
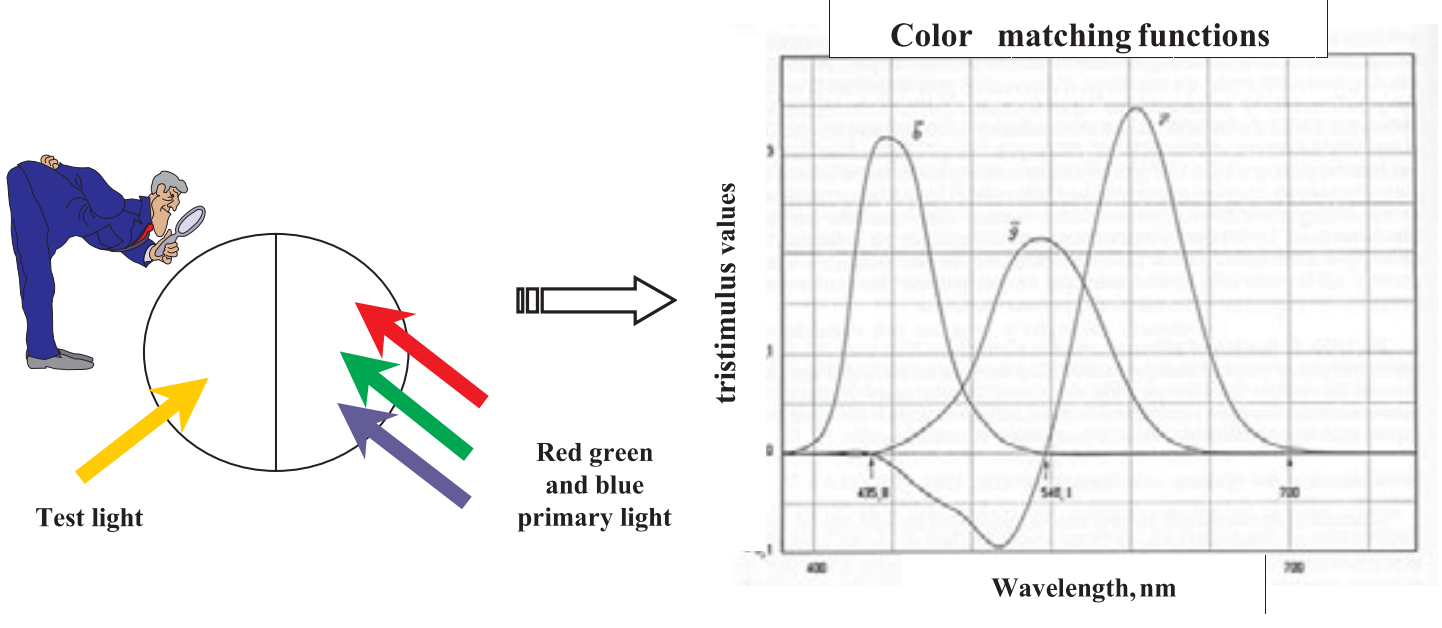

Fig. 6. Color matching functions obtained by color matching experiment (at a given wavelength, the values of the three functions $\bar{r}(\lambda), \bar{g}(\lambda), \bar{b}(\lambda)$ give the respective amounts of $R, G$ and $B$ primaries required to reproduce the perceived test color.) 
Color matching functions

( solid line : $2^{\circ}$ observer -dashed line : $10^{\circ}$ observer)

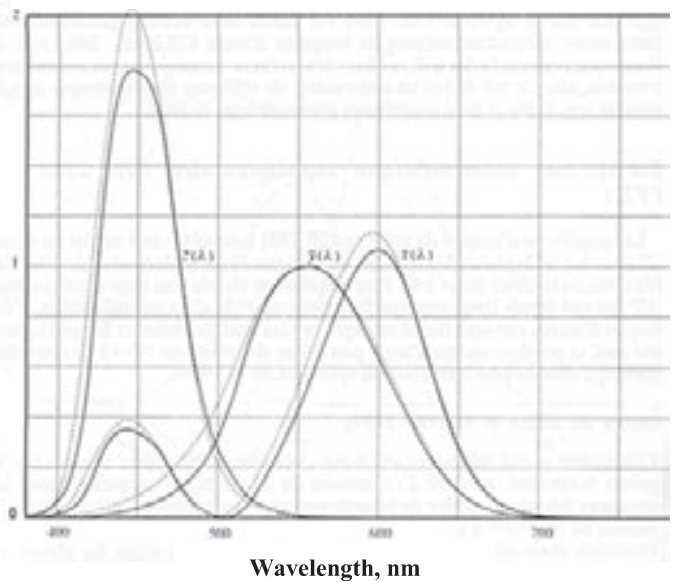

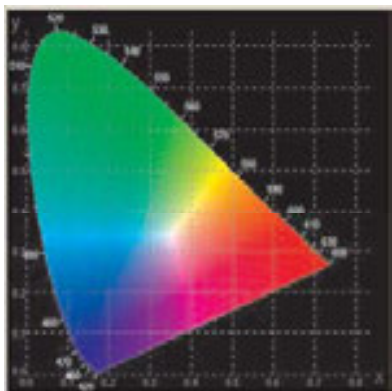

CIE 1931 (x,y) Chromaticity diagram (projection of the (color space on the $\mathrm{X}+\mathrm{Y}+\mathrm{Z}=1$ plane)

$$
\begin{aligned}
& x=\frac{X}{X+Y+Z} \\
& y=\frac{Y}{X=Y=Z} \\
& z=\frac{Z}{X=Y=Z}=1-x-y
\end{aligned}
$$

Fig. 7. $\bar{x}(\lambda), \bar{y}(\lambda), \bar{z}(\lambda)$ CIE standard color matching function: the three matching functions are positive - the area under the three matching functions is the same - The $\bar{y}(\lambda)$ function corresponds exactly to the luminous efficiency function of the eye.

As previously said, $\bar{r}(\lambda)$ shows negative values. This is why the CIE has mathematically defined positive observer matching functions $\bar{x}(\lambda), \bar{y}(\lambda), \bar{z}(\lambda)$ (Fig. 7). The corresponding $\mathrm{X}, \mathrm{Y}, \mathrm{Z}$ tristimuli values are always positive. Color matching functions depend on the viewing angle. The CIE defined accordingly two different standard observers corresponding to a maximum viewing angle of $2^{\circ}$ and of $10^{\circ}$, respectively.

The CIE X, Y, Z matching functions do not correspond to physical primaries. The (X, Y, Z) tristimuli values are obtained from Eq. 4:

$$
\left\{\begin{array}{l}
X=\int_{\lambda \min }^{\lambda \max -} x(\lambda) \cdot S(\lambda) \cdot d \lambda \\
Y=\int_{\lambda \min }^{\lambda \max -} y(\lambda) \cdot S(\lambda) \cdot d \lambda \\
Z=\int_{\lambda \min }^{\lambda \max -} \mathrm{Z}(\lambda) \cdot S(\lambda) \cdot d \lambda
\end{array}\right.
$$

The $(\mathrm{X}, \mathrm{Y}, \mathrm{Z})$ values can be obtained from $\mathrm{CIE}$ standard (R, G, B) trislimuli values from Eq. 5:

$$
\left[\begin{array}{c}
X \\
Y \\
Z
\end{array}\right]=\left[\begin{array}{lll}
2.7690 & 1.7518 & 1.1300 \\
1.0000 & 4.5907 & 0.0601 \\
0.0000 & 0.0565 & 5.5943
\end{array}\right] \bullet\left[\begin{array}{l}
R \\
G \\
B
\end{array}\right]
$$

CIE RGB and XYZ color spaces remain linearly related to the spectral distribution of color stimuli. ( $R$, $\mathrm{G}, \mathrm{B})$ and $(\mathrm{X}, \mathrm{Y}, \mathrm{Z})$ color spaces are not psychometric (i.e. non-uniform): when just noticeable visual differences are produced, the two corresponding points in $(R, G, B)$ or $(X, Y, Z)$ color spaces are not equi-spaced throughout the color space. In 1976, the CIE proposed two pseudo uniform color spaces called CIEL*a*b* (Fig. 8) and CIELuv. They have been mathematically designed in accordance to matching experiments on close colors. Some experimental work has been performed in very precise viewing conditions so that the results cannot be generalized to any other viewing contexts. Values are calculated from the $(\mathrm{X}, \mathrm{Y}, \mathrm{Z})$ color space in a non-linear way (Fig. 8). In these color spaces, the just noticeable difference is almost constant throughout the entire color space: an Euclidean distance corresponds to a color difference measurement (quantitative representation of the perceived color difference between a pair of colored samples); an equal distance corresponds approximativaly to an equal color difference. This color difference measurement is relevant when applied to close colors (what would be the meaning of a color difference between a green and a blue?): thus it is supposed to be perceptually consistent, from a perception point of view. 


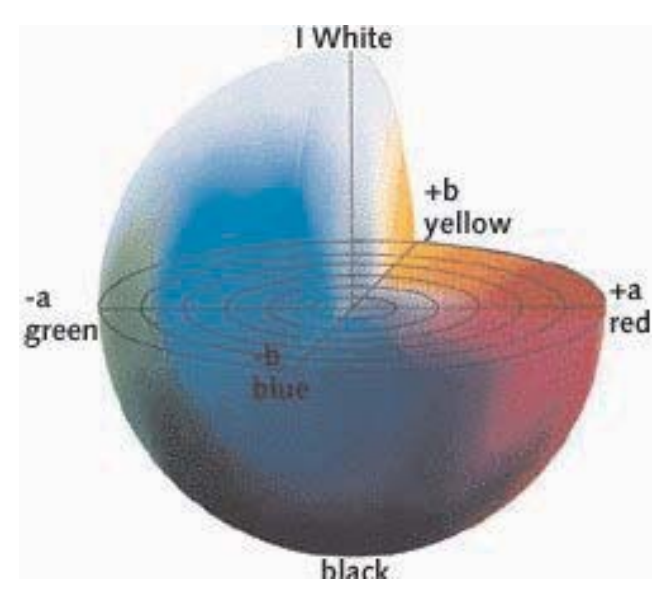

$$
\begin{aligned}
& L^{*}=116 f\left(Y / Y_{N}\right)-16 \\
& a^{*}=500\left[f\left(X / X_{N}\right)-f\left(Y / Y_{N}\right)\right] \\
& b^{*}=200\left[f\left(Y / Y_{N}\right)-f\left(Z / Z_{N}\right)\right] \\
& f(\alpha)=\left\{\begin{array}{c}
\alpha / 3 \\
7.787 \alpha+16 / 116
\end{array},\right. \text { otherwise } \\
& \text { with }\left(\mathrm{X}_{\mathrm{N}}, \mathrm{Y}_{\mathrm{n}}, \mathrm{Z}_{\mathrm{n}}\right) \text { tristimulus values of a white stimulus under a given } \\
& \text { standard illuminant }
\end{aligned}
$$

Fig. 8. CIEL $a^{*} b^{*}$ pseudo uniform color space.

As said above, the CIELab color space has been experimentally designed to measure the color difference of plain samples in a set of standard conditions. It separates the achromatic brightness information and the color information. In this color space, the hue is quantitatively defined as arctan (b/a) (angle between the color vector and the $+a$ axis) and the chroma corresponds to $\sqrt{a^{2}+b^{2}}$ (distance between the color locus and the midpoint). The brightness is represented vertically by $\mathrm{L}$, with values ranging from 0 (black) to 100 (white).

\section{COLOR IMAGE GRABBING}

There exist a wide range of imaging devices (see the example in Fig. 9) whose functions are image acquisition, image processing and image analysis. They are initially designed to quantify and display color images and not to measure color: in order to measure a color it remains to define a device independent color space. It is essential to take a system approach to digital color imaging in order to allow the rational use of color in a material characterization process; if color is considered as a measurable property, the acquisition system must be considered as a measuring apparatus and the user must control all the electronic settings.

\section{Image capture}

Image acquisition concerns the processing of electronic information from different sources (photoreceptors) that are not standardized (Giordanni and Madden, 1997; Laflaquière et al., 1998; Hardeberg, 1999). Most of image sensors in videocameras, scanners or digital photocameras are CCD-sensor (Charge Coupled Device). A CCD sensor is color blind: in order that it produced a color image, it is necessary to filter the incoming light. Different sources capture color stimuli in a different way. The spectral responsitivity of sensors differs among systems; of course none of them corresponds to CIE standard observer color-matching functions (Fig. 7).

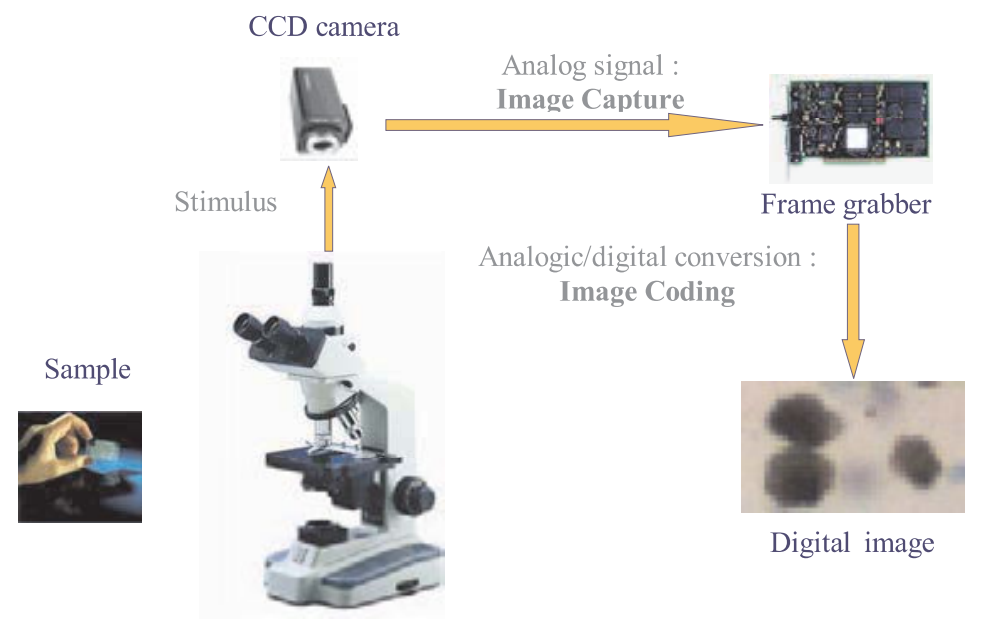

Fig. 9. Color imaging system. 
In order to permit the color management of an acquisition system, it is necessary to study output signals from the three video channels (Red, Green and Blue output signals). Depending on the goal of the performed study, it is more or less necessary to control the working of the acquisition system: when visual information is displayed for human observation, the acquisition and display device must be adjusted so that the image is realistic. In order to measure with a camera the color characteristics of a heterogeneous sample, both accuracy and invariability of the color capture must be studied. In a characterization process, if the studied element has a special spectral behavior in the visible range (color signature), the acquisition system must distinguish this specific signature.

So it is significant to analyse:

- The noise that corrupts signals: Dark current can be statistically estimated through the acquisition of a set of black images. The non uniform spatial response of a sensor can be compensated by using of an average image obtained from a white surface viewed under diffuse lighting conditions.

- Camera and digitizer board settings (gamma correction, white balance, profit, offset...) (Sangwine and Horne, 1998; Berns, 2000).

- The signal sampling and quantification.

- The sensor spectral responsitivity. It is important to analyse trichromatic capture of the original color stimulus through Charge Coupled Device sensors. The linearity of the sensor responses has to be analyzed.

A point of importance is that cameras can capture the color through physically realisable primaries (the $\mathrm{R}$ color matching function shows a negative part that cannot be reproduced by a sensor; a sensor is not able to give a negative response). Their spectral responsivities will never be shaped like CIE R, G, B color matching functions. The $\mathrm{R}, \mathrm{G}$ and $\mathrm{B}$ color filters used in $\mathrm{CCD}$ cameras are not standardized; they differ slightly with the filters required for color measurements (colorimeters). In others words, each acquisition system has a specific color gamut (range of colors that can be generated by mixing the chosen primary colors). Therefore, the color of two objects can be metameric with respect to camera sensors and non-metameric with respect to a human observer. When the acquisition process aims at obtaining a device-independent image, it is necessary to calibrate it in order to obtain deviceindependent color values. When the color is considered as the property to be measured on the image, it is necessary to calibrate colorimetrically the acquisition system: in order to determine how an image acquisition system actually sees color, it is required to compare sensors values and colorimetric values obtained on plain surfaces. This operation requires the capture of a color target (GretagMacbeth ColorChecker, Kodak Q60R1 target, IT8.7 Agfa target, ...) where all the values are measured with color instruments such as a spectrophotometer.

\section{Color coding}

The efficiency of a digital color imaging system depends partially on the coding of the processed color.

A color system or color space is altogether a means to systematically organize the set of all possible perceived colors. Many device-dependent and device independent color systems are proposed to the user. An universal color system does not exist. Generally color systems are three-dimensional spaces portraying the trichromatic properties of the human visual system. Each color space is designed to manage some specific properties of color. It is related to a color model generally based on specific primaries and specific viewing conditions: a color CRT monitor does not work with the same R, G and B primaries than a color video camera.

Color spaces can be classified in four categories:

- physiologically inspired systems based on primaries: (R, G, B), (Cyan, Magenta, Yellow color system used for printing), (CIEX, Y, Z used for color measurement and formulation), ...

- systems based on Luminance-Chrominance dissociation: (H, S, V Hue, Saturation, and Value color space used to select color from a color palette), (Y, I, Q space used by U.S. Commercial Color Television Broadcasting), (A, C1, C2) opponent model based on opponent primaries (yellow-blue and red-green for the chromatic information - black/white for the achromatic information)(Faugeras, 1979), ...

uniform systems (for visual perception): (CIEL*a*b* color space designed for determining perceptual color differences in some specific viewing conditions), Munsell color order system (Value, Chroma, Hue) (Munsell, 1969), ...

- color systems with independent axes specifically proposed for image processing (non correlated color components): (I1, I2, I3) (Ohta et al., 1980), ... 
Of course, each color space has its own advantages and drawbacks. For instance, color systems such as the $\mathrm{L}^{*} \mathrm{a}^{*} \mathrm{~b}^{*}$ are obtained through a non-linear transform in order to achieve perceptual uniformity. They lead to real number coding and non-uniform sampling of color space; some numerical instabilities may be encountered.

Effectiveness of color coding depends on how it is interpreted by the user. A color space must be selected in agreement with the concerned application (the relevant color information must be captured, and coded). As previously said, each imaging system has a specific color gamut. The corresponding color components are defined in a device-dependent way, and the device dependent color space is restricted to the numerical sampling of only a part of the natural color gamut (range of all possible colors).

\section{IMAGE PROCESSING AND ANALYSIS}

Once a numerical color image is captured and coded, the operator has to transform it in order to identify the relevant and the non-relevant information. An image may be viewed as a set of objects where each object presents a certain "visual homogeneity". This homogeneity is partly related to the radiometric attributes of the pixels and also partly related to some spatial considerations (Fig. 10).

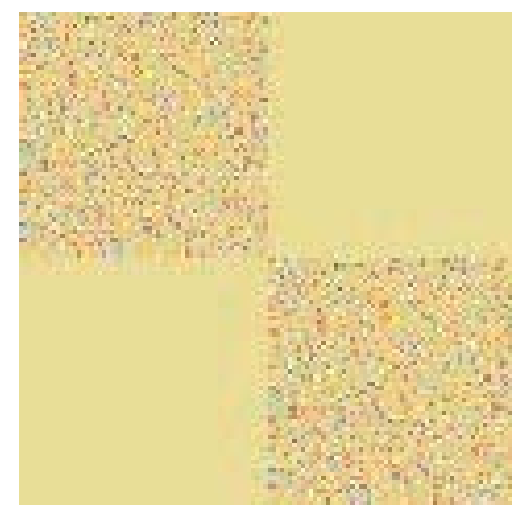

Fig. 10. Influence of radiometric attributes and spatial considerations on visual "homogeneity": observers will see four squares even if the two textured ones have a very important radiometric inhomogeneity.

\section{FILTERING AND SEGMENTING OF COLOR IMAGES}

Here, our intention is to draw the reader's attention on some general points of importance in filtering and segmenting color images (Lambert and
Macaire, 2000). A preliminary step of image processing consists in defining exactly what homogeneity is (in relation to the problem of characterization to be solved). In other words, it is necessary to define some geometric and color attributes that characterize the areas of interest in the image.

Filtering a color image aims at removing all nonrelevant color information while preserving the relevant color information that will be used in the segmentation.

The pre-processing step consists in filtering noise. The various filtering strategies applied to color images will not be detailed. Color images have specific characteristics compared to grey level images:

- Color images are multicomponent images. There is a correlation between the color components.

- It is possible to change the working color space according to the applied filters. As previously seen, each color space has some specific properties that may be used in a filtering process.

- It is significant to ensure the color integrity of the image and to avoid the appearance of false colors during the filtering step. This is all the more true when color is considered as a psychovisual property. Nevertheless, the false color syndrome is not as serious as it seems, a few false pixels within a multitude being not easily distinguishable.

- Scalar image filtering is based on ordering relations. To extend scalar methods to the color case, it is necessary to sort the color vectors corresponding to the color coordinates of every pixel.

Two approaches are possible in color image filtering (Chanussot, 1998):

- A marginal approach that considers each component independently. This approach ignores totally the correlation between color components.

- A vectorial approach where the image is globally processed (use of a partial or total ordering relation in a multidimensional space) (Goutsias, 1992; Chamussot and Lambert, 1998). The goal is to establish an ordering relation that respects the meaningful perceptual attributes of the image. At preent, there is no general answer to the false color problem. The first step in a filtering process consists in establishing a quantitative criteria (distance) to define and detect false colors of a filtered image in an appropriate color space. False colors must be defined with respect to the application.

The segmentation step consists in partitioning images in order to extract the meaningful information. 
The final result is a binary, or a labelled image. Two dual approaches are possible for segmenting a color image:

- The extraction of discontinuities. The color edge detection is based on gradient operators either in a monodimensional space or in a multidimensional space.

- The detection of homogeneous areas. Neighborhood-based segmentation analyses simultaneously spatial and color information in order to split an image in several regions. Segmentation can be based on an a priori knowledge of the color properties of the objects in the image. A very helpful preliminary step to image segmenting may consist in a change of the color space leading to a numerically more tractable representation of the relevant information (color coding in accordance with perceived color differences or with color contrasts). Again, the definition of homogeneous regions does not systematically correspond to close areas in the selected color space. Objects presenting a colortextured surface are rather difficult to extract.

Fig. 11 shows the results obtained with a segmentation process using the color attributes of the different mineral phases. In order to ease the segmentation and to guarantee the reproducibility of the characterization process, a change of color space has been first performed. The Lab color space has been chosen because it is a device independent color space. The different mineral phases have been colorimetrically characterized. For the considered material, the Lab space allows a segmentation by using successive threshold operations in the different color planes.

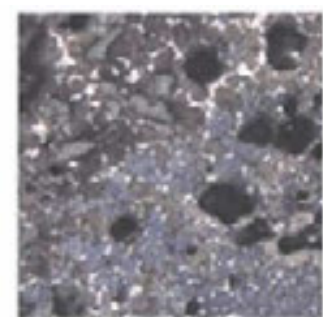

Initiale image $R G B$ color space

Plan L

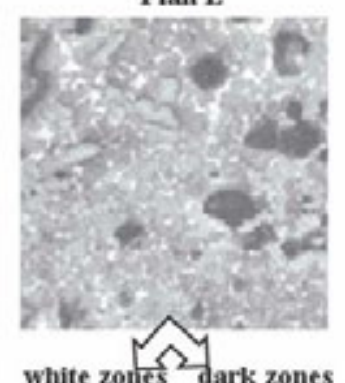

Plan a
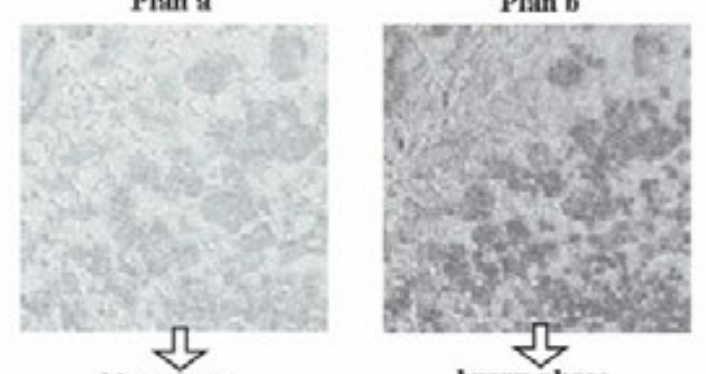

blue phase braun phase

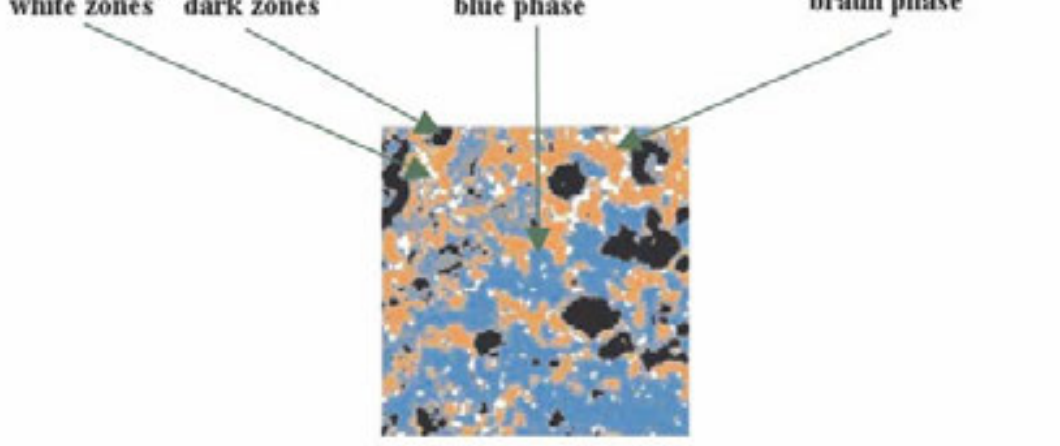

Fig. 11. Characterization of a mineral mixture - microscopic image - four phases (a blue one, a brown one, a grey one and a dark one). 


\section{Color image analysis}

Several industrial applications involving color image analysis already exist (Colot et al., 1998; Raffy et al., 1999; Hanbury and Gardieux, 2001). The quantitative analysis presented herein aims at characterizing color textures in terms of psychovisual impact on the end-user. In fact, sensorial analyses are now performed on plain colors, and do not describe random texture color variability in terms of perceived visual difference. It is necessary to define a system allowing the correspondence between perception and measurements on textured surfaces. Along that line, we perform a statistical and spatial analyses of the color attributes of the object under study. It is a necessary condition in order to manage correctly the color characteristics of the product. This study is a preliminary experiment allowing a) to define control protocols based on a sensorial approach, b) an automated monitoring of manufactured products with a color texture that is commercially used. Furthermore, it provides a basis for a virtual formulation of aspect related to a realistic image representation of the prototyped product (in a computer aided design process).

Fig. 12 presents two images of wood boards: (a) a massive wood board and (b) a MDF (medium density fibre) board. Of course, without any specification about illuminant, the specification of the object color by any tristimuli values is meaningless. The Acquisition has been performed under the same lighting conditions. The acquisition system has been calibrated in order to prevent any lighting drift: the color has been coded in the (X, Y, Z) color system (device independent) (De Valois, 1965). The objective of the analysis is to determine which relevant parameters can be obtained from images in the sense of a psychovisual color texture description. This is why color is ultimately expressed in the Lab system (Fig. 8) by introducing physical information concerning lighting conditions in the coding system (a non-uniform spatial response of the sensors measurement of Xn, Yn, $\mathrm{Zn})$. The observed variability in the a plane is partly due to a defective red channel acquisition in our acquisition system: the upper part of images shows systematically a reddish zone (Fig. 12). We did not correct this defect. The a plane, very sensitive to this artefact, is accordingly not studied.

The statistical analysis of the pixel color attributes in the two images shows that the mean values and standard deviations are very close, both for the $\mathrm{L}$ and $\mathrm{b}$ planes. However, their visual appearances differ. Massive wood board image highlights an alternation of clear stripes $v s$. dark ones due to the color of final wood (winter wood) and initial wood (spring wood) fibres. For the MDF, fibres are mixed and a plain appearance is perceived. Thus, relying on the sole statistics for texture color characterisation is here insufficient. This is why we consider below the analysis of the spatial distribution of color image attributes.

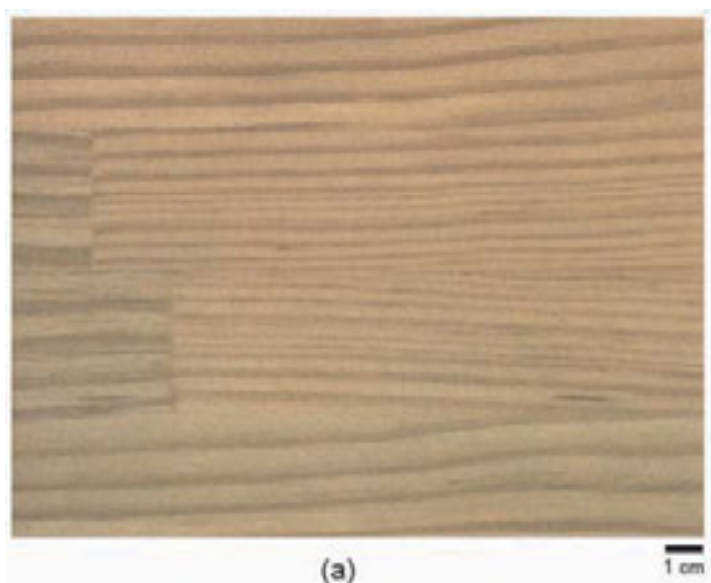

(a)

\begin{tabular}{l|cc|cc|cc}
\hline & \multicolumn{2}{|c|}{$\mathrm{L}$} & \multicolumn{2}{c|}{$\mathrm{a}$} & \multicolumn{2}{c}{$\mathrm{b}$} \\
\hline \multirow{3}{*}{ Massive wood } & Mean & Std deviation & Mean & Std deviation & Mean & Std deviation \\
MDF & 79,66 & 3,54 & 7,86 & 3,98 & 28,1 & 4,98 \\
& 77,79 & 1,33 & 6,29 & 3,69 & 24,03 & 4,21 \\
\hline
\end{tabular}

(c)

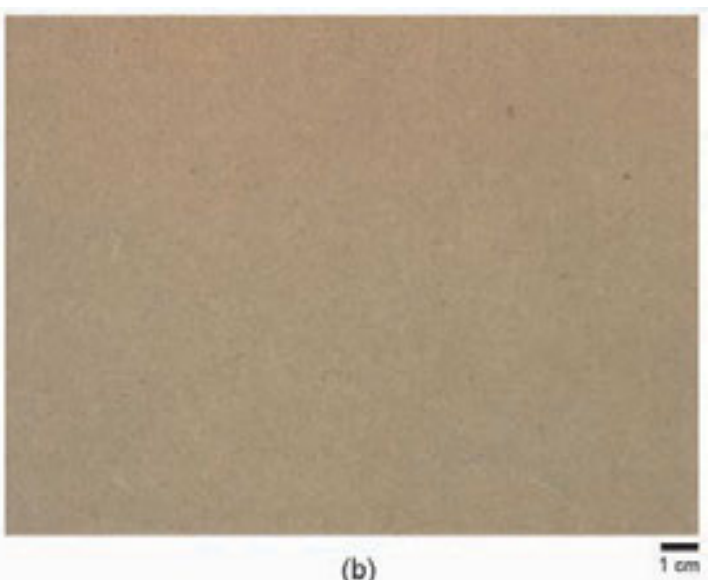

(b)

Fig. 12. Wood images: (a) massive wood board (b) MDF board (c) Statistics for L, a b values obtained through the calibration process 
The spatial analysis of the color information can be performed separately for every component of the color space (marginal approach): it consists in analyzing the variogram of every component. The variogram of an intrinsic random function is given by Eq. 6 (Matheron 1967; Armstrong and Carignan, 1997).

$$
\gamma(h)=\frac{1}{2} \operatorname{var}[Z(x+h)-Z(x)]
$$

For a fixed angle, it indicates how different the values become as the distance between sample points increases.

The marginal approach does not give complete information on the color texture because the observer does not distinguish in its analysis information on $\mathrm{L}$, independently from $\mathrm{a}$ or $\mathrm{b}$. In the Lab color space, the Euclidean distance corresponds to perceived color differences. It is the ACD (average color difference) that informs about the color structure recognized as a non-random texture by the observer. Spatial analysis can then be performed from a more global point of view. That consists in studying average color differences as a function of the distance between pixels along one direction (Eq. 7). It indicates the variation of the colorimetric difference when the distance between sample points increases.

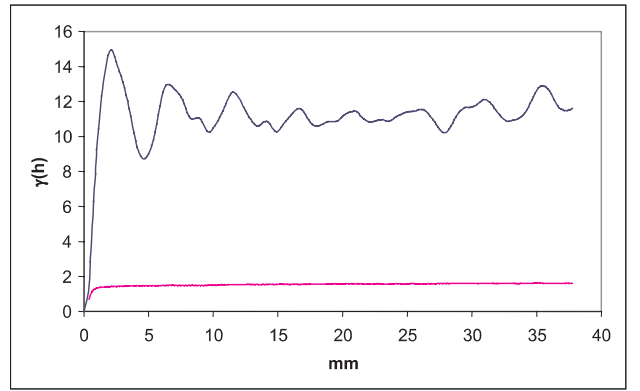

(a)

$$
\begin{aligned}
& A C D(h)=\frac{1}{n} \sum_{i=1}^{n} \Delta E(i, i+h) \\
& \text { with } \Delta E(i, i+h)= \\
& \qquad \sqrt{\left(L_{i}-L_{i+h}\right)^{2}+\left(a_{i}-a_{i+h}\right)^{2}+\left(b_{i}-b_{i+h}\right)^{2}}
\end{aligned}
$$

The analysis of the variogram of the $\mathrm{L}$ and $\mathrm{b}$ components (Figs. 13a-b), along a direction perpendicular to the wood fibres, highlights differences in the spatial repartition of $\mathrm{L}$ and $\mathrm{b}$. The calculated ACDs (Fig. 13c) express aspect differences between the two images. As the variograms of $L$ and $b$, the ACD function for the massive wood board shows periodic undulations (with a period of about $5 \mathrm{~mm}$ ) expressing alternance of dark colored final wood and light colored initial wood. Here again (Fig. 13c), the average width of the stripes is $5 \mathrm{~mm}$. Note that for the MDF, the variogram presents a nugget effect (Matheron, 1967) and is consistent with a purely random model with no correlation between sample points, whatever the lag is. The nugget effect may be caused either by measurement errors or by the microstructure i.e., a color component of the image whose range is smaller than the smallest discrete lag at which the variogram is computed. In our example, the use of the ACD function, lower than 8 for MDF, is obviously relevant in a quantitative approach to perception of color texture.

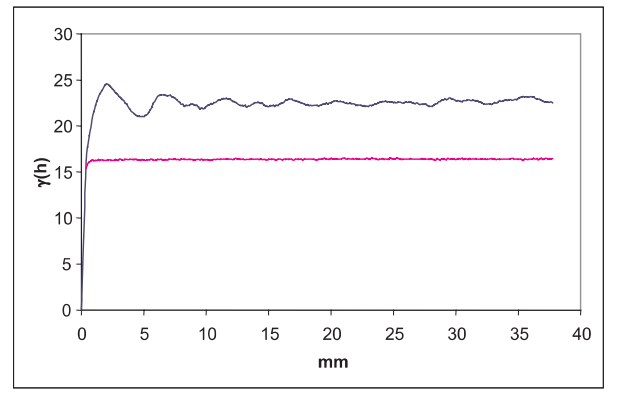

(b)

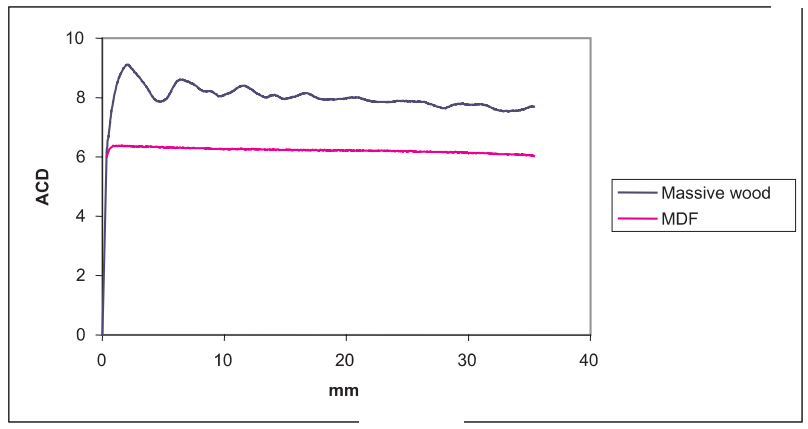

(c)

Fig. 13. Semi-variogram along the vertical direction (a) on $L$ values (b) on $b$ values (c) average color difference. 
In image simulation, the $\mathrm{L}, \mathrm{a}$ and $\mathrm{b}$ values must be chosen one by one. Then, it becomes interesting to evaluate the impact on visual perception of each component of the color space. To define which of $\mathrm{L}$ or $b$ carries the most relevant information in terms of visual perception, we have created a texture model obtained from the interpretation of the experimental variogram on the massive wood board. Then, we varied separately the values of $L$ and $b$, first in the light strata and then, in the dark strata of the model (Fig. 14). At last, we have compared the original image and the modified ones.

a)

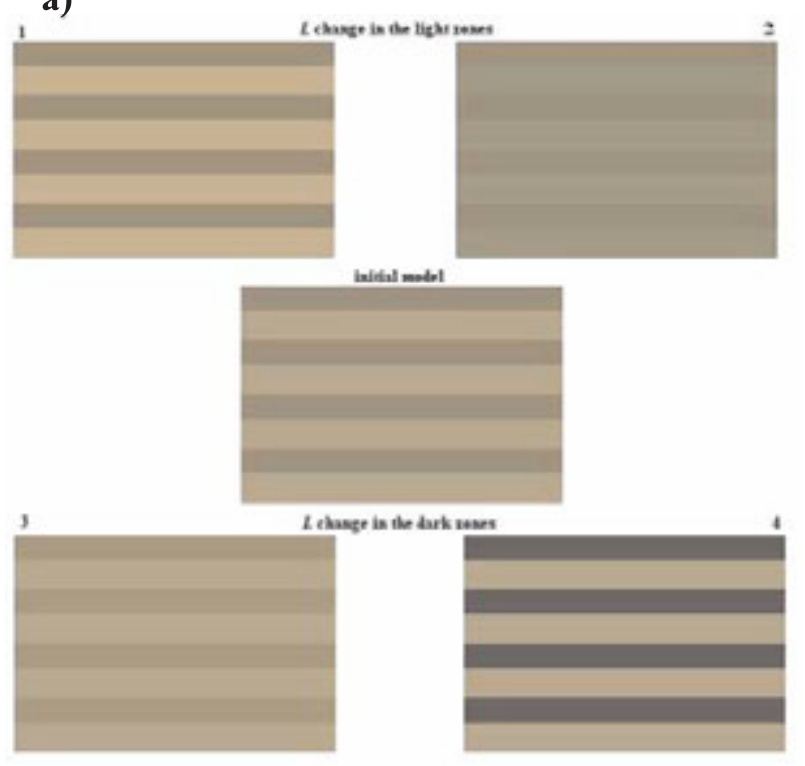

b)

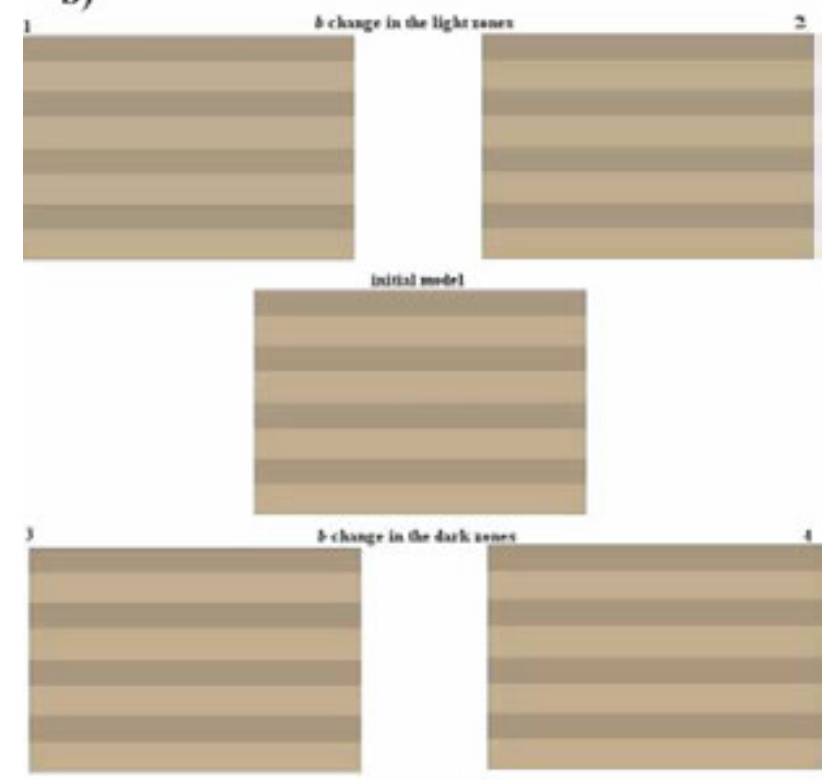

Fig. 14. Evaluation of $L$ and $b$ changes - for each zone $L$ and $b$ values are successively and separately increased and decreased - a) corresponds to $L$ changes - b) corresponds to $b$ changes.
Fig. 14 shows that the observer is more sensible to a variation of $\mathrm{L}$ than $\mathrm{b}$ in the color texture.

\section{CONCLUSION}

The use of color images in the characterization of materials is an important challenge for scientific and industrial applications. A pluri disciplinary approach of the problem is necessary in order to correctly manage color information. Going from grey scale images to color images emphasizes the fact that image acquisition systems play a central role in the success of such an undertaking. Another very important point concerns the sample preparation and the applied viewing conditions. It is very convenient to work with an a priori knowledge of the physicochemical properties of the objects under study. The characterization of visual properties of materials is a new and interesting field of investigation. It leads to a general thinking about perception quantification: in an hedonist approach of materials, it is very interesting to wonder and predict how one material will "live» in its own environment. The formulation of visual aspect in material industry is a promissing way.

\section{ACKNOWLEDGEMENTS}

This paper owes much to the multidisciplinary work carried out by the members of all of the group «Propriétés Psycho Sensorielles» of the Centre des Matériaux de Grande Diffusion de L'Ecole des Mines d'Alès.Much is also due to the work performed in the framework of the GdR ISIS (Groupe de Recherche Information, Signal, Images et viSion du CNRS) and in particular to a book under preparation for DUNOD («Traitement et Analyse des images couleur. La couleur pour mieux voir !»).

\section{REFERENCES}

Armstrong M, Carignan J (1997). Géostatistique linéaire, application au domaine minier. Les Presses de l'Ecole des Mines.

Barnes CS, Weis J, Shewell SK (1999). Chromatic induction with remote chromatic contrast varied in magnitude, spatial frequency and chromaticity. Vision Research 39:3561-74.

Bedat V (1998). Aspects psychovisuels de la perception des couleurs: application au codage d'images couleur fixes avec compression de l'information. $\mathrm{PhD}$ thesis, IRESTE, Université de Nantes.

Berns RS (2000). Principles of colour technology. New York: John Wiley \& Sons. 
Bowmaker JK, Dartnall HJA (1980). Visual Pigments of rods and cones in a human retina. J Physiol, 298:501-11.

Bozec-Garay H (2000). Paramètres morphogranulométriques et comportement optique des matériaux divisés. $\mathrm{PhD}$ Thesis, Ecole des Mines de Saint-Etienne.

Chamussot J, Lambert P (1998). Total ordering based on space filling curves for multivalued morphology". Proc. $4^{\text {th }}$ International Symposium on Mathematical Morphology and its Applications. Amsterdam, The Netherlands, 51-8, June 1998.

Chanussot J (1998). Approches vectorielles ou marginales pour le traitement d'images multi-composantes". PhD Thesis, Université de Savoie.

Colot O, Devinoy R, Sombo A, de Brucq D (1998). A colour image processing method for mellanoma detection. Lectures Notes in Computer Science 1496:562-9.

De Valois L (1965). Analysis and coding of color vision in the primate visual system. Cold Spring Harbor Symposium on Quantitative Biology 30:471-92.

D'Zmura M, Rinner O, Gegenfurtner K (2000). The colors behind a transparent filter. Perception 29:1169-84.

Faugeras OD (1979). Digital color image processing within the framework of a human visual model. IEEE Transaction on Acoustics, Speech and Signal Processing 27(4):380-93.

Giordanni EJ, Madden TE (1997). Digital color management. Addison-Wesley, 576p.

Goutsias J (1992). Morphological transformation of image sequences: a latice theory approach. Proc SPIE, vol. 1769. Image Algebra and Morphological Image Processing 3, San Diego, 306-17, 1992

Hanbury A, Gardieux F (2001). A quantitative description of wood texture. Proc Int Conf Quality Control by Artificial Vision (QCAV'2001), 451-60, 2001.

Hardeberg JY (1999). Acquisition et reproduction d'images couleur: approches colorimétrique et multispectrale. $\mathrm{PhD}$ Thesis, Ecole Nationale Supérieure des Télécomunications, ENST 99 E 021.
Judd DB, Wyszecki G (1975). Color in Business, Science and Industry. Third Edition. New York: Jonh Wiley \& Sons.

Laflaquière $\mathrm{Ph}$, Lafon $\mathrm{D}$, Eterradossi O, Slangen $\mathrm{P}$ (1998). Characterization of color texture - CIEL*a*b* calibration of CCD device. Int Symp on Electronic Image Capture and Publishing, Europto Series 98, "Image Capture \& Scanning"session, communication $n^{\circ}$ 3409-20. Zurich 18-20 May.

Lambert P, Macaire L (2000). Filtering and segmentation: the specificity of colour images, in CGIP'2000: $1^{\text {st }}$ Int Conf on color in Graphics and Image Processing, Saint Etienne, France, october 2000, 57-71.

Longère P, Shewell SK, Knoblauch K (2000). Invest Ophtalmol Vis Sci, Suppl., 41:S805.

Matheron G (1967). Les variables régionalisées et leur estimation. Masson et Cie Eds. Paris.

Munsell A (1969). A Grammar of Color. Van Nostrand, New York.1969.

Ohta YI, Kanade T, Sakai T (1980). Color information for region segmentation. Comp Graph Image Proc 13:22241.

Raffy G, Marty-Mahé P, Ollivier M, Marchal P (1999). Real-time detection of appearance defects of turkey carcasses by color histogram analysis. In SPIE Machine Vision Application in Industrial Inspection VII, Electronic Imaging'99, 3652, San-Jose, USA, 1999.

Roorda A, Williams DR (1999). The arrangement of the three cone classes in the living human eye. Nature 397:520-2.

Sangwine SJ, Horne REN (1998). The colour image processing handbook. Chapman \& Hall.

Sève R (1996). Physique de la couleur. Masson, 334 p.

Wyszeski G, Stiles WS (1982). Color science: concepts and methods, Quantitative Data and Formulae. New York: Jonh Wiley \& Sons. 